\title{
Presence of magnetic excitations in SmFeAsO
}

\author{
Jonathan Pelliciari, ${ }^{1, \text { a) }}$ Marcus Dantz, ${ }^{1}$ Yaobo Huang, ${ }^{1,2}$ Vladimir N. Strocov, ${ }^{1}$ Lingyi Xing, ${ }^{2}$ \\ Xiancheng Wang, ${ }^{2}$ Changqing Jin, ${ }^{2,3}$ and Thorsten Schmitt ${ }^{1, b)}$ \\ ${ }^{1}$ Swiss Light Source, Paul Scherrer Institut, CH-5232 Villigen PSI, Switzerland \\ ${ }^{2}$ Beijing National Laboratory for Condensed Matter Physics, Institute of Physics, \\ Chinese Academy of Sciences, P. O. Box 603, Beijing 100190, China \\ ${ }^{3}$ Collaborative Innovation Center for Quantum Matters, Beijing 100871, China
}

(Received 2 August 2016; accepted 5 September 2016; published online 19 September 2016)

\begin{abstract}
We measured dispersive spin excitations in $\mathrm{SmFeAsO}$, a parent compound of $\mathrm{SmFeAsO}_{1-\mathrm{x}} \mathrm{F}_{\mathrm{x}}$ and one of the highest temperature superconductors of $\mathrm{Fe}$ pnictides $\left(\mathrm{T}_{\mathrm{C}} \approx 55 \mathrm{~K}\right)$. We determine the magnetic excitations to disperse with a bandwidth energy of ca $170 \mathrm{meV}$ at $(0.47,0)$ and $(0.34$, 0.34 ), which merges into the elastic line approaching the $\Gamma$ point. Comparing our results with other parent $\mathrm{Fe}$ pnictides, we show the importance of structural parameters for the magnetic excitation spectrum, with small modifications of the tetrahedron angles and As height strongly affecting the magnetism. Published by AIP Publishing. [http://dx.doi.org/10.1063/1.4962966]
\end{abstract}

Since the discovery of high temperature superconductivity $(\mathrm{SC})^{1}$ in $\mathrm{LaFeAsO}_{1-\mathrm{x}} \mathrm{F}_{\mathrm{x}}$, the number of $\mathrm{Fe}$-based compounds has quickly boosted and new families differing in structure and stoichiometry have been discovered and synthesized, the most common being $\mathrm{SmFeAsO}$ (1111), $\mathrm{BaFe}_{2} \mathrm{As}_{2}$ (122), NaFeAs (111), and FeSe (11) (see Refs. 2 and 3 for extended reviews). Ubiquitous FeAs layers, composed of $\mathrm{FeAs}_{4}$ tetrahedrons, are separated by spacers, which differ from family to family. Generally, the parent compounds are antiferromagnetically ordered, and SC emerges upon hole, electron, or isovalent doping, with the dopants either in the FeAs or in the spacing layer. ${ }^{2,3}$ The phase diagram, characterized by antiferromagnetism and SC, is similar to other unconventional superconductors, such as the cuprates and heavy fermion systems where a magnetic-mediated superconducting pairing mechanism has been proposed. ${ }^{4,5}$ Similarly, such a scenario has been further extended from these systems to Fe pnictides. ${ }^{4,5}$ In this framework, residual antiferromagnetic (AF) fluctuations are expected to be strong and possibly lead to a superconducting phase. Moreover, the structural/nematic transition at $\mathrm{T}>\mathrm{T}_{N}$ has been associated with spin excitations. ${ }^{2,3,6}$ Thus, the detection of these AF fluctuations within several families with different structures is of vital importance for a complete understanding of Fe pnictides.

The effect of magnetism on the electronic structure has been measured by Angle Resolved Photo-Emission Spectroscopy (ARPES) through the detection of a kink in the band structure, ${ }^{7-9}$ which has been ascribed to electron-boson coupling with the bosonic candidate being of magnetic nature. However, ARPES represents an indirect spectroscopy technique to characterize magnetism which has to be characterized by means of techniques sensitive to spin excitations, such as neutron scattering ${ }^{10-14}$ and/or X-ray scattering. ${ }^{15,16}$ Neutron scattering experiments confirmed the presence of sizable magnetic moments in $\mathrm{Fe}$ pnictides (on the order

\footnotetext{
a) jonathan.pelliciari@psi.ch

b) thorsten.schmitt@psi.ch
}

of $\left.\approx 1 \mu_{B}\right)^{2,3,10}$ with few exceptions, such as $\mathrm{NaFeAs}$ that shows lower ordered magnetic moment. ${ }^{2,3}$ On the dynamical side, spin wave-like excitations were also observed in the AF phases of several compounds by inelastic neutron scattering (INS) and Resonant Inelastic X-Ray Scattering (RIXS). ${ }^{10,14-19}$ The importance of spin fluctuations has been further confirmed by their persistence within the superconducting phase, ${ }^{10,15-19}$ even though a conclusive picture of their role is still under development.

1111 crystals are known for polar cleaving naturally which makes the interpretation of surface sensitive spectroscopic data (such as ARPES) difficult because of the mixing of surface states with bulk states. ${ }^{9,20-23}$ Moreover, the growth of suitable crystals for INS is challenging and this complicates the measurements of high energy spin excitations $(>90 \mathrm{meV})$, where INS would provide plenty of information. ${ }^{10-12,24}$ These drawbacks can be minimized by employing RIXS, which has been previously employed in the detection of high energy spin excitations in NaFeAs and $\mathrm{BaFe}_{2} \mathrm{As}_{2} \mathrm{Fe}$ pnictides. ${ }^{15,16}$ Moreover, thanks to the high refocusing (beam spot of $5 \times 20 \mu \mathrm{m}^{2} \mathrm{~V} \times \mathrm{H}$ at the ADRESS beamline of the Swiss Light Source) and flux obtainable at the sample for modern beamlines ${ }^{25}$ the amount of sample required for these investigations is on the order of tens of milligrams and crystals of the size $150 \times 200 \mu \mathrm{m}$ can now be successfully studied, even measuring down to a single layer of material. ${ }^{26}$

In this letter, we report on the measurement of high energy spin excitations in $\mathrm{SmFeAsO}$, a parent compound of the 1111 series. We identify dispersing magnetic excitations ranging up to an energy of $170 \mathrm{meV}$ at $(0.47,0$ r.l.u.) which merge into the elastic line at the $\Gamma$ point. Similar behavior is detected along the diagonal direction, where spin excitations disperse to $160-170 \mathrm{meV}$ at $(0.34,0.34$ r.l.u. $)$ and decrease in energy moving towards the $\Gamma$ point. The spin excitation bandwidth has a value similar to $\mathrm{BaFe}_{2} \mathrm{As}_{2}(\approx 190 \mathrm{meV})$ but higher than $\mathrm{NaFeAs}(\approx 150 \mathrm{meV})$. We correlate the bandwidth of magnetic excitations with the structure of the FeAs 4 tetrahedron, in particular, the height of As with respect to the 
Fe layer $\left(\mathrm{h}_{\mathrm{FeAs}}\right)$. The detection of high energy spin excitations in this parent compound confirms that magnetic excitations are universal within the parent Fe pnictides.

Single crystals of $\mathrm{SmFeAsO}$ have been grown by the flux method, using $\mathrm{NaAs}, \mathrm{SmAs}, \mathrm{Fe}_{2} \mathrm{O}_{3}, \mathrm{Fe}$, and $\mathrm{As}$ powder as starting materials. The precursor NaAs has been obtained by mixing $\mathrm{Na}$ lump and As powder, which had been sealed in an evacuated titanium tube and sintered at $650{ }^{\circ} \mathrm{C}$ for $10 \mathrm{~h}$. SmAs has been prepared by mixing $\mathrm{Sm}$ pieces and As powder, sealed in a evacuated $\mathrm{Ti}$ tube, and sintered at $700^{\circ} \mathrm{C}$ for $20 \mathrm{~h}$. The stoichiometric amounts of $\mathrm{NaAs}, \mathrm{SmAs}, \mathrm{Fe}_{2} \mathrm{O}_{3}, \mathrm{Fe}$, and As powder have been weighed to achieve an element ratio of NaAs:SmFeAsO = 20:1. The mixture has been ground thoroughly and put into an alumina crucible and sealed in an $\mathrm{Nb}$ crucible under 1 atm of Argon gas, which was then sealed in an evacuated quartz tube. Finally, the mixture was heated to $1100^{\circ} \mathrm{C}$ and cooled slowly down to $700{ }^{\circ} \mathrm{C}$ at a rate of $5^{\circ} \mathrm{C} / \mathrm{h}$ to grow the single crystals.

The samples were mounted with the $a b$ plane perpendicular to the scattering plane and the $c$ axis lying in it (sketch in Fig. 1(a)) and post-cleaved in situ at a pressure better than $2.0 \times 10^{-10}$ mbar. The directions studied are $(1,0)$ and $(1,1)$ according to the orthorhombic unfolded crystallographic notation. $^{27}$ We use the convention of $1 \mathrm{Fe}$ per unit cell. All the measurements were carried out at $10 \mathrm{~K}$. X-ray Absorption Spectra (XAS) and RIXS experiments were performed at the ADRESS beamline of the Swiss Light Source, Paul Scherrer Institute, Villigen PSI Switzerland. ${ }^{25,28}$ XAS spectra were measured in Total Fluorescence Yield (TFY). The RIXS spectrometer was set to a scattering angle of $130^{\circ}$ and the incidence angle on the sample's surface was varied to change the in plane momentum transferred $(q)$ from $(0,0)$ to (0.47, 0 r.l.u.) (relative lattice units expressed in $\mathrm{q} / / \cdot \mathrm{a} / 2 \pi$ ) and from $(0,0)$ to $(0.34,0.34$ r.l.u.) as shown in Fig. 1(a). All measurements are recorded in grazing incidence configuration. The total energy resolution was $110 \mathrm{meV}$, measured by means of elastic scattering from a carbon-filled acrylic tape.

We measured $\mathrm{Fe} \mathrm{L}_{2,3}$ XAS spectra for the two crystallographic orientations at $15^{\circ}$ incidence angle and $\pi$ polarization. Fig. 1(b) displays the XAS spectrum of the $(1,0)$ orientation. The XAS along $(1,1)$ (not displayed) is analogue to the $(1,0)$ direction. The spectrum is composed of a broad peak centered at $707 \mathrm{eV}$, typical of metallic systems containing Fe. ${ }^{15,16,29,30}$ The incident energy for RIXS was tuned at the main $\mathrm{Fe}-\mathrm{L}_{3}$ peak. In
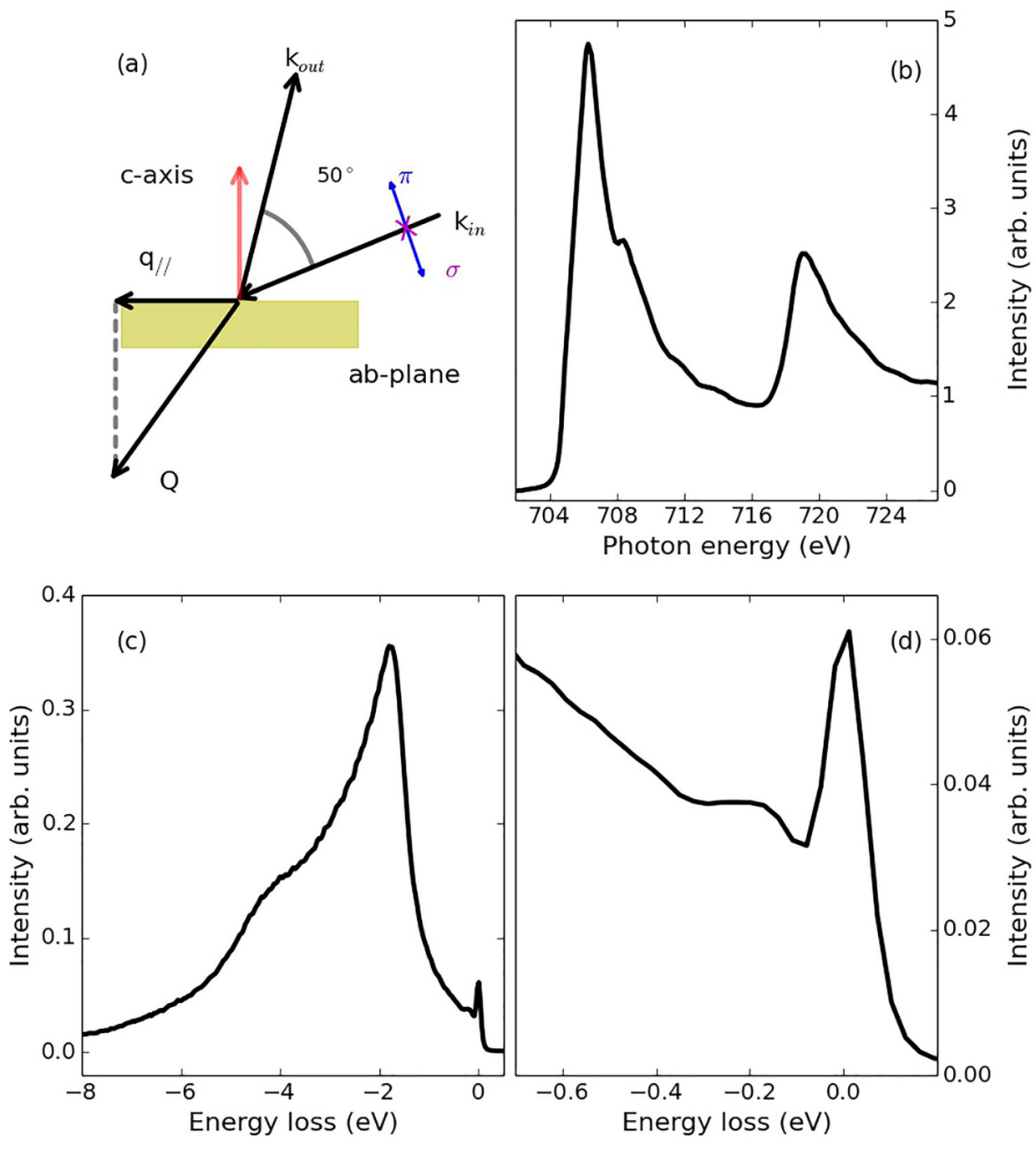

FIG. 1. (a) Experimental geometry of RIXS experiments. (b) XAS spectrum of $\mathrm{SmFeAsO}$ at $15^{\circ}$ between the incoming beam and sample surface along (1,0) with $\pi$ polarization. (c) RIXS spectrum of $\mathrm{SmFeAsO}$ at $15^{\circ}$ between the incoming beam and sample surface along $(1,0)$. Polarization was set to $\pi$ and the incident energy was tuned on the maximum of the XAS spectrum shown in (b). (d) Zoom into the low energy region of (c). Temperature is $10 \mathrm{~K}$ for all the spectra. 


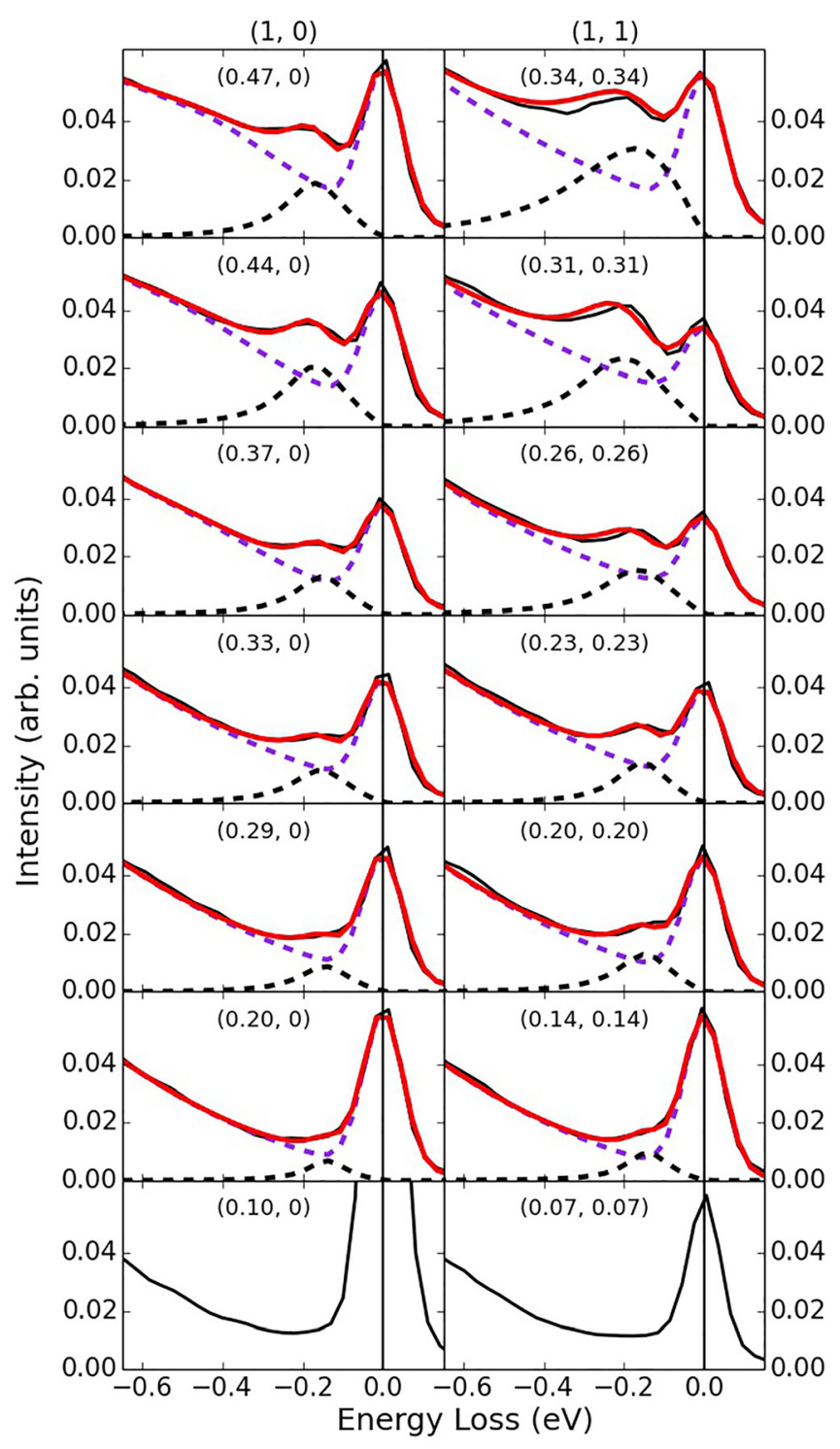

FIG. 2. Momentum dependence of RIXS spectra along $(0,0) \rightarrow(0.47,0)$ and $(0,0) \rightarrow(0.34,0.34$ r.l.u. $)$ for $\mathrm{SmFeAsO}$. Spectra were recorded in $\pi$ polarization at the maximum of the $\mathrm{Fe} \mathrm{L}_{3}$ absorption edge at $10 \mathrm{~K}$. Experimental data are shown as the black solid line, fitting of the background and elastic line are the purple dashed line, and the magnetic peaks are depicted as the black dashed line for every spectrum. The total fitting is depicted as the red solid line. At low $\mathrm{q}_{/ /}$a fitting is unreliable, so no fitting is attempted.

Fig. 1(c), an exemplary RIXS spectrum of SmFeAsO at $(0.47,0)$ is shown. The main line in this spectrum resembles emission from metallic systems with a broad asymmetric peak displaying a maximum at around $-2 \mathrm{eV}$ in energy loss $\left(\mathrm{h} \nu_{\text {out }}-\mathrm{h} \nu_{\text {in }}\right),{ }^{15,16,29,31}$ arising from resonant emission of itinerant electrons. In contrast to doped $1111,{ }^{32}$ but in agreement with other $\mathrm{Fe}$ pnictides, the emission line is not showing any sharp features ascribable to dd-excitations. ${ }^{15,16,29,30}$ This confirms the moderate electronic correlations of $\mathrm{Fe}$ pnictides contrasting with $\mathrm{Fe}$ chalcogenides where dd-excitations have been observed employing hard X-ray RIXS. ${ }^{33}$

On the low energy side of the RIXS spectra at $(0.47,0)$ and $(0.34,0.34)$, we observe a peak emerging from the background having an energy of $170 \mathrm{meV}$ as illustrated in Figs. 1(d) and 2. This peak moves towards the elastic line when the in-plane momentum transferred is decreased and at low $\mathrm{q}_{/ /}$it merges into the elastic line. The energy range and the dispersive nature of this mode resemble the behavior of spin excitations detected by RIXS in $\mathrm{BaFe}_{2} \mathrm{As}_{2}{ }^{15}$ and $\mathrm{NaFeAs}$ (measurements shown in supplementary material). We fit the background, the elastic and the magnetic line in agreement with Refs. 15, 16, and 31 and plot the results in Fig. 2. At low values of $\mathrm{q} / /$, we do not attempt any fitting procedure because of the high overlap between the elastic and the magnetic peak. However, we believe that an estimation of a rough energy range is still possible. In Fig. 3(a), we show the dispersion curve arising from this fitting procedure as black dots with error bars together with the dispersion curves extracted from $\mathrm{BaFe}_{2} \mathrm{As}_{2}{ }^{15}$ and $\mathrm{NaFeAs}$ measured in the same experimental conditions (see the raw data and the fittings of NaFeAs in the supplementary material).

Figure 3(a) illustrates the dispersion relation of magnetic excitations for $\mathrm{SmFeAsO}$ (black dots with error bars), NaFeAs (green dots with error bars), and $\mathrm{BaFe}_{2} \mathrm{As}_{2}$ (red dots with error bars) along $(1,0)$ and $(1,1)$. Clearly, there is a renormalization of the magnetic bandwidth between $\mathrm{BaFe}_{2} \mathrm{As}_{2}$, that shows the highest energy (190 meV $\pm 20 \mathrm{meV}), \mathrm{SmFeAsO}$ displaying an intermediate energy $(170 \mathrm{meV} \pm 20 \mathrm{meV})$, and $\mathrm{NaFeAs}$ having the lowest bandwidth $(150 \mathrm{meV} \pm 20 \mathrm{meV})$ as summarized in Fig. 3(c). This trend is in qualitative agreement with the decreasing values of magnetic moment from $\mathrm{BaFe}_{2} \mathrm{As}_{2}$ to $\mathrm{SmFeAsO}$ and NaFeAs. ${ }^{2,3,10,14,34}$ However, a quantitative comparison between ordered magnetic moment and spin excitations is not straightforward and is beyond the goal of this paper. The relevance of the structural parameters for SC and magnetism has been widely discussed in Refs. 2, 3, and 14, with $\mathrm{h}_{\mathrm{FeAs}}$ and the angle of the tetrahedron formed by $\mathrm{FeAs}_{4}$ as the main possible parameters affecting $\mathrm{T}_{C}$ and $\mathrm{T}_{N}$. Since there is no SC in these parent compounds, in Fig. 3(b) we show only the values of $\mathrm{T}_{N}$ and how they correlate with $\mathrm{h}_{F e A s}{ }^{2,3,14}$ In detail, $\mathrm{h}_{F e A s}$ increases from $1.358 \AA$ in $\mathrm{BaFe}_{2} \mathrm{As}_{2}$ to $1.37 \AA$ in $\mathrm{SmFeAsO}$ and then to $1.416 \AA$ in NaFeAs, whereas $\mathrm{T}_{N}$ decreases $2,3,10,14,34$ as well as the magnetic bandwidth measured in our experiments and outlined in Fig. 3(c). This highlights the importance of the structure for the spin excitations in Fe pnictides as well as the expected role of structural deformations due to doping, pressure or even defects in phase transitions to nematic and/or superconducting phases.

In conclusion, we measured the spin excitation spectrum of the SmFeAsO, a parent compound of the 1111 series, bypassing the polar cleaving problem. A comparison with other parent Fe pnictides, measured in the same experimental configuration, shows that the bandwidth of spin waves is slightly renormalized to lower values in $\mathrm{SmFeAsO}$ compared to $\mathrm{BaFe}_{2} \mathrm{As}_{2}$ but higher than NaFeAs. Here, we have illustrated how the structure, in particular, $\mathrm{h}_{F e A s}$, influences the magnetic properties of $\mathrm{Fe}$ pnictides possibly triggering most of the modifications. We claim that, if such structural modifications can affect the magnetism and bandwidth, then the perturbations provided by doping and/or pressure might lead to instabilities such as Cooper pairing and/or nematicity, not 

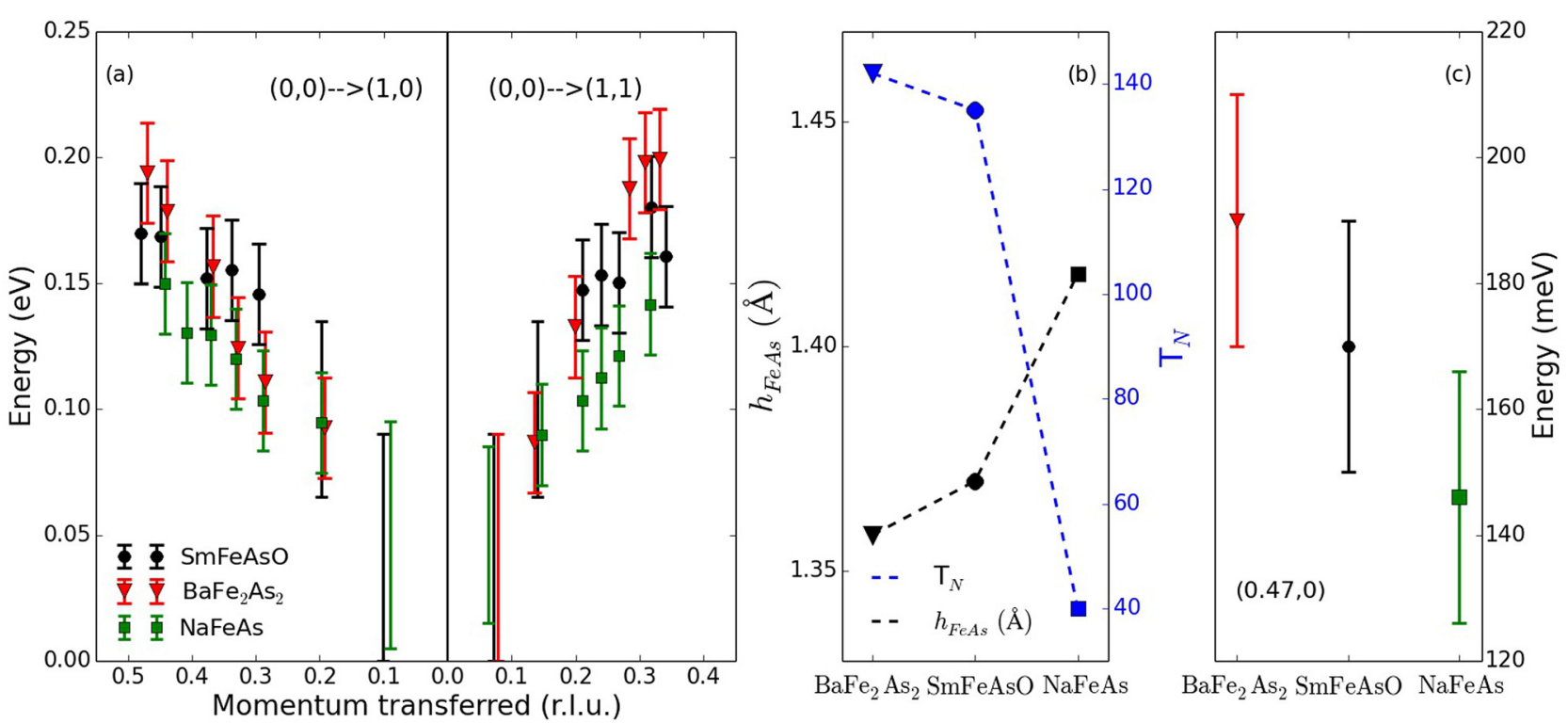

FIG. 3. (a) Dispersion of spin excitations in $\mathrm{BaFe}_{2} \mathrm{As}_{2},{ }^{15} \mathrm{SmFeAsO}$, and $\mathrm{NaFeAs}$ detected along $(1,0)$ and $(1,1)$ extracted from RIXS experiments at $10 \mathrm{~K}$. Close to $(0,0)$, we show only the error bar without marker because the peak of magnetic excitations and the elastic line are overlapped and a determination of the energy is unreliable (see text). (b) Comparison of $\mathrm{T}_{N}$ and $\mathrm{h}_{\mathrm{FeAs}}$ in $\mathrm{BaFe}_{2} \mathrm{As}_{2}, \mathrm{SmFeAsO}$, and $\mathrm{NaFeAs}$ from Refs. 2 and 3. (c) Summary of the spin excitations energy at $(0.47,0)$.

triggered by electronic effects alone but by structural effects as well.

See supplementary material for the raw RIXS data of $\mathrm{NaFeAs.}$

J.P. and T.S. acknowledge financial support through the Dysenos AG by Kabelwerke Brugg AG Holding, Fachhochschule Nordwestschweiz, and the Paul Scherrer Institut. Part of this research has been funded by the Swiss National Science Foundation through the D-A-CH programme (SNSF Research Grant No. 200021 L 141325). Experiments have been performed at the ADRESS beamline of the Swiss Light Source at Paul Scherrer Institut. The work at IOP-CAS was supported by NSF and MOST through research projects.

${ }^{1}$ Y. Kamihara, T. Watanabe, M. Hirano, and H. Hosono, J. Am. Chem. Soc. 130, 3296 (2008).

${ }^{2}$ G. R. Stewart, Rev. Mod. Phys. 83, 1589 (2011).

${ }^{3}$ D. C. Johnston, Adv. Phys. 59, 803 (2010).

${ }^{4}$ D. J. Scalapino, Rev. Mod. Phys. 84, 1383 (2012).

${ }_{5}^{5}$ A. Chubukov, Annu. Rev. Condens. Matter Phys. 3, 57 (2012).

${ }^{6}$ R. M. Fernandes, A. V. Chubukov, and J. Schmalian, Nat. Phys. 10, 97 (2014).

${ }^{7}$ P. Richard, T. Sato, K. Nakayama, S. Souma, T. Takahashi, Y.-M. Xu, G. F. Chen, J. L. Luo, N. L. Wang, and H. Ding, Phys. Rev. Lett. 102, 047003 (2009).

${ }^{8}$ H. Liu, G. F. Chen, W. Zhang, L. Zhao, G. Liu, T.-L. Xia, X. Jia, D. Mu, S. Liu, S. He, Y. Peng, J. He, Z. Chen, X. Dong, J. Zhang, G. Wang, Y. Zhu, Z. Xu, C. Chen, and X. J. Zhou, Phys. Rev. Lett. 105, 027001 (2010). ${ }^{9}$ L. X. Yang, B. P. Xie, B. Zhou, Y. Zhang, Q. Q. Ge, F. Wu, X. F. Wang, X. H. Chen, and D. L. Feng, J. Phys. Chem. Solids 72, 460 (2011).

${ }^{10}$ P. Dai, Rev. Mod. Phys. 87, 855 (2015).

${ }^{11}$ J. M. Tranquada, G. Xu, and I. A. Zaliznyak, J. Magn. Magn. Mater. 350, 148 (2014)

${ }^{12}$ M. Fujita, H. Hiraka, M. Matsuda, M. Matsuura, J. M. Tranquada, S. Wakimoto, G. Xu, and K. Yamada, J. Phys. Soc. Jpn. 81, 011007 (2012).

${ }^{13}$ D. S. Inosov, C. R. Phys. 17, 60-89 (2016).
${ }^{14}$ C. Zhang, L. W. Harriger, Z. Yin, W. Lv, M. Wang, G. Tan, Y. Song, D. Abernathy, W. Tian, T. Egami, K. Haule, G. Kotliar, and P. Dai, Phys. Rev. Lett. 112, 217202 (2014).

${ }^{15}$ K.-J. Zhou, Y.-B. Huang, C. Monney, X. Dai, V. N. Strocov, N.-L. Wang, Z.-G. Chen, C. Zhang, P. Dai, L. Patthey, J. van den Brink, H. Ding, and T. Schmitt, Nat. Commun. 4, 1470 (2013).

${ }^{16}$ J. Pelliciari, Y. Huang, T. Das, M. Dantz, V. Bisogni, P. O. Velasco, V. N. Strocov, L. Xing, X. Wang, C. Jin, and T. Schmitt, Phys. Rev. B 93, 134515 (2016).

${ }^{17}$ H. Luo, Z. Yamani, Y. Chen, X. Lu, M. Wang, S. Li, T. A. Maier, S. Danilkin, D. T. Adroja, and P. Dai, Phys. Rev. B 86, 024508 (2012).

${ }^{18}$ H. Luo, X. Lu, R. Zhang, M. Wang, E. A. Goremychkin, D. T. Adroja, S. Danilkin, G. Deng, Z. Yamani, and P. Dai, Phys. Rev. B 88, 144516 (2013).

${ }^{19}$ M. Wang, C. Zhang, X. Lu, G. Tan, H. Luo, Y. Song, M. Wang, X. Zhang, E. A. Goremychkin, T. G. Perring, T. A. Maier, Z. Yin, K. Haule, G. Kotliar, and P. Dai, Nat. Commun. 4, 2874 (2013).

${ }^{20}$ S. de Jong, Y. Huang, R. Huisman, F. Massee, S. Thirupathaiah, M. Gorgoi, F. Schaefers, R. Follath, J. B. Goedkoop, and M. S. Golden, Phys. Rev. B 79, 115125 (2009).

${ }^{21}$ H. Eschrig, A. Lankau, and K. Koepernik, Phys. Rev. B 81, 155447 (2010).

${ }^{22}$ L. X. Yang, B. P. Xie, Y. Zhang, C. He, Q. Q. Ge, X. F. Wang, X. H. Chen, M. Arita, J. Jiang, K. Shimada, M. Taniguchi, I. Vobornik, G. Rossi, J. P. Hu, D. H. Lu, Z. X. Shen, Z. Y. Lu, and D. L. Feng, Phys. Rev. B 82, 104519 (2010).

${ }^{23}$ C. Liu, Y. Lee, A. D. Palczewski, J.-Q. Yan, T. Kondo, B. N. Harmon, R. W. McCallum, T. A. Lograsso, and A. Kaminski, Phys. Rev. B 82, 075135 (2010).

${ }^{24}$ M. Ramazanoglu, J. Lamsal, G. S. Tucker, J.-Q. Yan, S. Calder, T. Guidi, T. Perring, R. W. McCallum, T. A. Lograsso, A. Kreyssig, A. I. Goldman, and R. J. McQueeney, Phys. Rev. B 87, 140509 (2013).

${ }^{25}$ V. N. Strocov, T. Schmitt, U. Flechsig, T. Schmidt, A. Imhof, Q. Chen, J. Raabe, R. Betemps, D. Zimoch, J. Krempasky, X. Wang, M. Grioni, A. Piazzalunga, and L. Patthey, J. Synchrotron Radiat. 17, 631 (2010).

${ }^{26}$ M. P. M. Dean, R. S. Springell, C. Monney, K. J. Zhou, J. Pereiro, I. Božović, B. Dalla Piazza, H. M. Rønnow, E. Morenzoni, J. van den Brink, T. Schmitt, and J. P. Hill, Nat. Mater. 11, 850 (2012).

${ }^{27}$ J. T. Park, D. S. Inosov, A. Yaresko, S. Graser, D. L. Sun, P. Bourges, Y. Sidis, Y. Li, J.-H. Kim, D. Haug, A. Ivanov, K. Hradil, A. Schneidewind, P. Link, E. Faulhaber, I. Glavatskyy, C. T. Lin, B. Keimer, and V. Hinkov, Phys. Rev. B 82, 134503 (2010).

${ }^{28}$ G. Ghiringhelli, A. Piazzalunga, C. Dallera, G. Trezzi, L. Braicovich, T. Schmitt, V. N. Strocov, R. Betemps, L. Patthey, X. Wang, and M. Grioni, Rev. Sci. Instrum. 77, 113108 (2006). 
${ }^{29}$ E. Z. Kurmaev, J. A. McLeod, N. A. Skorikov, L. D. Finkelstein, A. Moewes, Y. A. Izyumov, and S. Clarke, J. Phys.: Condens. Matter 21, 345701 (2009).

${ }^{30}$ W. L. Yang, A. P. Sorini, C.-C. Chen, B. Moritz, W.-S. Lee, F. Vernay, P. Olalde-Velasco, J. D. Denlinger, B. Delley, J.-H. Chu, J. G. Analytis, I. R. Fisher, Z. A. Ren, J. Yang, W. Lu, Z. X. Zhao, J. van den Brink, Z. Hussain, Z.-X. Shen, and T. P. Devereaux, Phys. Rev. B 80, 014508 (2009).

${ }^{31}$ J. N. Hancock, R. Viennois, D. van der Marel, H. M. Rønnow, M. Guarise, P.-H. Lin, M. Grioni, M. Moretti Sala, G. Ghiringhelli, V. N.
Strocov, J. Schlappa, and T. Schmitt, Phys. Rev. B 82, 020513 (2010).

${ }^{32}$ T. Nomura, Y. Harada, H. Niwa, K. Ishii, M. Ishikado, S. Shamoto, and I. Jarrige, Phys. Rev. B 94, 035134 (2016).

${ }^{33}$ H. Gretarsson, T. Nomura, I. Jarrige, A. Lupascu, M. H. Upton, J. Kim, D. Casa, T. Gog, R. H. Yuan, Z. G. Chen, N.-L. Wang, and Y.-J. Kim, Phys. Rev. B 91, 245118 (2015).

${ }^{34}$ Iron-Based Superconductivity, Springer Series in Materials Science Vol. 211, edited by P. D. Johnson, G. Xu, and W.-G. Yin (Springer International Publishing, Cham, 2015). 